had higher injury rates while older workers lost more days following falls. Rates of paid lost days associated with FFH decreased over time, but there was not a consistent decline in mean lost days per fall.

Conclusions Falls from height (FFH) continue to cause significant morbidity and mortality across the construction industry. The observed patterns are consistent with decreased FFH for several years surrounding the Washington Vertical Fall Arrest Standard (1991); the decline exceeds those seen in injury rates overall in this large construction cohort. While crude rates of FFH have continued to decline, they are not as substantial as the declines seen for other types of injuries. The patterns could reflect a variety of things including more global efforts designed to control risk (site planning, safety accountability) and changes in reporting practices.

\section{A LONGITUDINAL STUDY OF THE OCCUPATIONAL TONER EXPOSURE ON INFLAMMATION AND ALLERGIC BIOMARKERS}

H K Kitamura, Hata, Mizuno, Ogami, Higashi. Institute of Industrial Ecological Science, Kitakyushu, Japan

\subsection{6/oemed-2013-101717.230}

Objectives We have been conducting a cohort study since 2003 to examine the health effects caused by exposure to toner. The aim of this study is to evaluate the relationship between toner exposure and biomarkers from the results of a 7-year follow up.

Methods The subjects were 1,504 Japanese male workers aged below 50 in 2003. We divided toner-handling works into 5 categories, and then carried out personal exposure measurements on randomly selected workers in each category every year. We also surveyed the toner-handling work category through a self-administered questionnaire every year. Based on the results of the $1^{\text {st }}$ personal exposure measurements, toner dust levels were classified into 3 toner-exposed groups: high $\left(>=0.15 \mathrm{mg} / \mathrm{m}^{3}\right)$, medium $\left(=<0.02 \mathrm{mg} / \mathrm{m}^{3}, 0.15 \mathrm{mg} / \mathrm{m}^{3}<\right)$, and low $(0.02 \mathrm{mg} /$ $\mathrm{m}^{3}<$ ). Individual workers were classified into 3 toner-exposed groups each year based on the measured toner dust levels and toner-handling work category answered in the questionnaire. Workers who were not engaged in toner-handling work were defined as the non-exposed group. We measured C-reactive protein (CRP) and Immunoglobulin $\mathrm{E}$ (IgE) in serum and 8hydroxy-2'-deoxyguanosine (8-OHdG) in urine for biomarkers. Generalised Estimating Equations (GEE)was applied to examine the relationship between toner exposure and the biomarkers. The biomarkers were used as dependent variables and tonerexposed groups, smoking habits, allergic diseases, and age were used as independent variables.

Results None of the toner-exposed groups showed significant increases in any of the biomarkers in comparison with the nonexposed group. On the other hand, we found that $\operatorname{IgE}$ and 8OHdG significantly increased in current smokers compared to never smokers.

Conclusions This study suggests that the health effects of occupational toner exposure may less than those of smoking, and that the possibility of toner exposure to induce inflammation and allergy is quite low.

\section{INCREASED CARDIOVASCULAR MORTALITY AFTER METHYL CHLORIDE EXPOSURE}

\section{R Rafnsson, Kristbjornsdottir. University of Iceland, Reykjavik, Iceland}

\subsection{6/oemed-2013-101717.231}

Objectives Methyl chloride leakage from a refrigerator occurred on board an Icelandic fishing vessel in the year 1963. The exposure lasted four days and one man died during the fishing trip. Many of the crew members were hospitalised due to different neurological symptoms and signs, and had not recovered completely several years later. The aims were to study long-term mortality.

Methods This is a cohort study with external references. Five referents were randomly selected from registries of seamen and officers matched to each crew member according to age and employment status. Follow up was through record linkage of personal identifier with nationwide mortality registry. Hazard ratios (HR) and 95\% confidence intervals (CI) were estimated in Cox proportional hazards model adjusted for age and employment.

Result The intoxicated crew eligible for follow up were 20 deckhands and 7 officers, the reference group counted 100 deckhands and 35 officers. Followed up to end of 2010, 14 of the exposed deckhands had died and 6 of the officers versus 49 deckhands and 26 officers among the reference group. The HR for all causes of death was 2.10 (95\% CI 1.28-3.46). For all cardiovascular events HR was 2.06 (95\% CI 1.024.15), for acute coronary heart disease HR was 3.12 (95\% CI 1.11-8.78), for cerebrovascular diseases HR was 5.35 (95\% CI 1.18-24.35), and for suicide HR was 13.76 (95\% CI 1.18160.07).

Conclusions The follow up of the methyl chloride exposed cohort showed increased mortality due to cardiovascular diseases after 47 years. After the intoxication the suicide cases had developed severe depressions that were considered to be related to the exposure. The use of the personal identifiers and the comprehensive population registries strengthen the study. Comparison to non exposed group of the same occupations indirectly control for potential confounders including social class, occupational experience, lifestyle factors, diet, smoking, and alcohol use.

\section{STRIATAL PATHOLOGY ASSOCIATED WITH CHRONIC MANGANESE EXPOSURE}

${ }^{1} \mathrm{~B}$ A Racette, ${ }^{2}$ Gonzales, ${ }^{3}$ Nelson, ${ }^{2}$ Checkoway, ${ }^{2}$ Zhang. ${ }^{~}$ Washington University School of Medicine, St. Louis, United States of America; ${ }^{2}$ University of Washington, Seattle, United States of America; ${ }^{3}$ University of the Witwatersrand, Johannesburg, South Africa

\subsection{6/oemed-2013-101717.232}

Objective The purpose of this study was to investigate the neuropathology of chronic $\mathrm{Mn}$ exposure in South African Mn miners.

Methods We performed a neuropathologic study of eight deceased Mn miners and ten deceased, non-Mn reference miners, none of whom had a diagnosis of a $\mathrm{Mn}$ - related clinical syndrome. We compared mean cell density in the caudate, putamen, and globus pallidus interna and externa in Mn miners with non-Mn miners, using GFAP to immunostain astrocytes, CD68 to immunostain microglia, and MAP-2 to immunostain neurons.

Results There were no significant differences in age, race or gender between $\mathrm{Mn}$ and non-Mn miners. There was a trend toward a higher mean (standard error) pallidal index in the Mn miners 
[124.3 (3.9)] compared to the non-Mn miners [113.1 (5.1)] (p $=0.11$ ). Mn miners had lower mean (standard error) neuron density in the caudate [203.1(24.9) cells per high powered field (hpf)] compared to non-Mn miners [276.9 (22.3) cells per hpf] $(\mathrm{p}=0.016)$. Mn miners also had lower astrocyte density in the caudate [181 (22.0) cells per hpf] and putamen [225.6 (28.5) cells per hpf] than non $\mathrm{Mn}$ miners [caudate 252.9 (19.6) cells per hpf and putamen 300.9 (25.4) cells per hpf] ( $p=0.011$ and $\mathrm{p}=0.024$, respectively). There were no significant differences in microglial cell density or in astrocyte, microglia or neuron cell counts in the globus pallidus between the two groups. There were no HIV-defining pathologies and no microglial nodules in any of the miners.

Conclusions This study demonstrates that chronic Mn exposure is associated with selective toxicity to striatal astrocytes and caudate neurons. We speculate that the initial neurotoxic injury in humans with chronic $\mathrm{Mn}$ exposure involves the astrocytes and that neuronal injury may be secondary to loss of astrocytes.

\section{THREATS AND VIOLENCE AT THE WORKPLACE AND THE RISK OF DEVELOPING ANXIETY SYMPTOMS AND DEPRESSION}

1) F T Thomsen, ${ }^{2}$ Mikkelsen, ${ }^{3}$ Kaergaard, ${ }^{3}$ Kolstad, ${ }^{4}$ Hansen, ${ }^{2}$ Bonde, ${ }^{3}$ Andersen, ${ }^{3}$ Grynderup, ${ }^{5}$ Kaerlev, ${ }^{6}$ Mors, ${ }^{7}$ Rugulies. ${ }^{1}$ Bispebjerg Hospital, Copenhagen, Denmark; ${ }^{2}$ Department of Occupational and Environmental Medicine, Bispebjerg Hospital, Copenhagen, Denmark; ${ }^{3}$ Danish Ramazzini Centre, Herning, Denmark; ${ }^{4}$ Department of Public Health, University of Copenhagen, Copenhagen, Denmark; ${ }^{5}$ Centre for National Clinical Databases South, Odense University Hospital, Odense, Denmark; ${ }^{6}$ Centre for Psychiatric Research, Aarhus University Hospital, Aarhus, Denmark; ${ }^{7}$ National Research Centre for the Working Environment, Copenhagen, Denmark

\subsection{6/oemed-2013-101717.233}

Objectives Workplace threats and violence are common within certain professions. In the European countries, 5\% of workers report having been subjected to violence. The health consequences are both physical and mental. This study analyses if repeated threats and violence or serious violence may lead to an increased risk of anxiety symptoms and depression.

Methods In 2007 and 2009 we examined 3224 civil servants employed in hospitals, schools, social centres etc. Exposure at baseline was self-reported number of times the last 12 months subjected to threats or non-serious physical violence or to mobility- or life-threatening violence. In 2007 and 2009, anxiety symptoms were scored (range 0-4) with the Symptom Check List and clinical depression with a psychiatric interview (SCAN). Cases of anxiety symptoms (score $>2$ ) and clinical depression at baseline were excluded. We used logistic regression analyses with estimates adjusted for potential confounders.

Results In 2007, 1079 (34\%) reported that they had been subjected to threats or non-serious physical violence 1-5 times the last year and $170(5 \%)$ more than 5 times. Fifty-nine (2\%) had been subjected to mobility- or life-threatening violence. 173 developed symptoms of anxiety and 62 clinical depression. The adjusted odds ratios (ORs) for incident anxiety symptoms because of threats or non-serious violence 1-5 times were 1.69 (95\% confidence interval 1.18-2.42) and more than 5 times $2.53(1.34-4.77)$. The corresponding ORs for incident clinical depression were $1.32(0.73-2.38)$ and 1.85 (0.71-4.83), respectively. There were too few cases among the exposed to analyse for the effect of mobility- or life-threatening violence.
Conclusions Exposure to threats and non-serious violence predicted anxiety symptoms. The risk increased with increasing number of episodes. The same pattern was found for depression though not significant. The results indicate the importance of considering the less serious but more frequent episodes when planning prevention.

\section{Session: 2. Exposure assessment I}

\section{CHARACTERISATION OF WORK TASKS AND EXPOSURES TO CLEANING AND DISINFECTING CHEMICALS IN HEALTHCARE OCCUPATIONS}

${ }^{1}$ MA Virji, 'LeBouf, ${ }^{2}$ Saito, 'Liang, 'Stefaniak, 'Stanton, 'Humann, ${ }^{1}$ Henneberger. ${ }^{1}$ National Institute for Occupational Safety and Health, Morgantown, United States of America; ${ }^{2}$ University of Texas Health Science Center at Tyler, Tyler, United States of America

\subsection{6/oemed-2013-101717.234}

Objectives Cleaning and disinfecting products have been identified as important risk factors for asthma, and are used extensively in healthcare; however, quantitative measurements of these etiologic agents are not well characterized. The objective of this study was to characterize personal exposure to cleaning and disinfecting compounds and quantify the frequency and duration of cleaning tasks performed in healthcare occupations. Methods Exposure assessments were conducted for volatile organic compounds (VOCs) at 5 hospitals targeting 13 healthcare occupations. A wide range of specific VOCs $(n=15)$ were quantified and an additional 97 VOCs were identified but not quantified.

Results The geometric mean (GM) concentrations for total VOCs were highest among nursing assistants, licensed practical nurses and medical equipment preparers (GM range: 4367-3809 $\mathrm{ppb}$ ), followed by respiratory therapists, pharmacy technicians, registered nurses, floor strippers/waxers, dental assistants and housekeepers (GM range: 2119-1501 ppb); the geometric standard deviations (GSD) varied from 1.8 to 7.5 across occupations. The GM and GSD of specific VOCs were also variable across occupations. The average amount of time per day spent on cleaning tasks using cleaning and disinfecting products also varied by occupation with medical equipment preparers, housekeepers, floor strippers/waxers and licensed practical nurses spending the most time (range: 165-110 minutes/day), followed by endoscopy technicians and dental assistants (range: 70-60 minutes/ day); the remaining occupations spent on average $<15$ minutes/ day on cleaning tasks.

Conclusions The chemical agents, levels of total and specific VOCs, and cleaning-task durations varied between- and withinoccupations indicating that task may be an important exposure determinant.

\section{AIRBORNE AND INTERNAL EXPOSURE TO CHROMIUM AMONG WELDERS}

${ }^{1} \mathrm{~A}$ L Lotz, ${ }^{1}$ Weiss, ${ }^{1}$ Pesch, ${ }^{2}$ Van Gelder, ${ }^{2}$ Hahn, ${ }^{1}$ Brüning. 'Institute for Prevention and Occupational Medicine of the DGUV (IPA), Bochum, Germany, ${ }^{2}$ Institute for Occupational Safety and Health of the DGUV (IFA), Sankt Augustin, Germany

\subsection{6/oemed-2013-101717.235}

Objectives The objective of this analysis was to investigate levels and determinants of exposure to respirable and urinary 